\title{
Wobbly Hedgehog Syndrome (WHS): a review of knowledge and some practical suggestions about differential diagnosis
}

\author{
JAKUB J. RUSZKOWSKI
}

\begin{abstract}
Institute of Zoology, Department of Animal Anatomy, Faculty of Veterinary Medicine and Animals Sciences, Poznan University of Life Sciences, Wojska Polskiego 71C, 60-625 Poznań
\end{abstract}

\section{Ruszkowski J. J. \\ Wobbly Hedgehog Syndrome (WHS): a review of knowledge and some practical suggestions about differential diagnosis}

Summary

With increase in popularity of African pygmy hedgehogs kept as pets, veterinarians have a chance of getting more information about housing requirements and diseases of the species. Wobbly Hedgehog Syndrome (WHS) and various neoplasms have been detected frequently. Diagnostic procedures for differentiating nervous system diseases in hedgehogs are still being developed and the prevalence of different diseases seems to be depended on the genetic origin of an animal. The main goal of the article was to review of published data, compare the prevalence of diseases and pathological findings. There is still a need to establish good diagnostic schemes for a check-up examination to detect early stage of the diseases.

Keywords: WHS, review, pets, hedgehog, atelerix, erinaceus, myelinopathy

There is a growing interest in exotic pets worldwide. One of them is the African Pygmy Hedgehog (Atelerix albiventris). Hedgehogs are member of the order Eulipotyphla, nocturnal, solitary animals (19). The majority of the scientific and clinical-based knowledge about hedgehogs and their diseases come from North America $(2,4,7,8,15,17)$ and Asia $(13,14,16)$ but there are also European reports $(9,11)$. In the last two decades, the number of scientific papers about diseases in pet hedgehogs increased. Neoplasms and Wobbly Hedgehog Syndrome (WHS) seem to be frequently reported causes of death $(2,4,7-9,11-13,16)$. WHS and neoplasms of the central nervous system are hard to diagnose in living animals, which makes diagnostic procedures harder. However, other diseases involving respiratory, digestive and cardiac systems were also reported $(2,4,7,9,11,16,17)$.

The main goal of the review was to summarize current knowledge, which is not comprehensive, see if there are any predisposing factors that can be eliminated to increase the lifespan of the hedgehogs and propose possible diagnostic procedures.

Neoplasms and WHS with a prevalence (in the USA) of $29 \%$ to $51.5 \%(8)$ and $10 \%(7)$, respectively, are the main reported causes of death in pet hedgehogs.
WHS is a neurologic, incurable, progressive disease of the African Pygmy Hedgehog. A similar, if not the same disease was reported in European Hedgehog (Erinaceus europaeus). Those hedgehogs also had lesions in central nervous system: demyelination in the brain and spinal cord, but there was also an inflammatory response in meninges, which was absent in hedgehogs with WHS (15). So far WHS can be diagnosed only by post-mortem examination of samples from central nervous system (CNS). Neoplasms of CNS with a prevalence of $1.6 \%$ (12) were reported with clinical symptoms very similar to those occurring in WHS $(2,4,9,11-13)$. In patients with both WHS and CNS neoplasms diagnosed by post-mortem examination, the symptoms included ataxia, hindlimb paresis, falling to one side paralysis, seizures with dysphagia in late stages of the disease. Moreover, the average age of animals in both cases was similar: 2.5 to 3.5 years. Both CNS neoplasms and WHS showed tendencies to occur in family lines, which may suggests inherited factor linked with the disease $(7,16)$.

The primary histologic lesions of hedgehogs with WHS were status spongiosus of the white matter of the cerebrum, cerebellum and brain stem, myelin loss and degeneration $(2,7)$, and as reported in European 


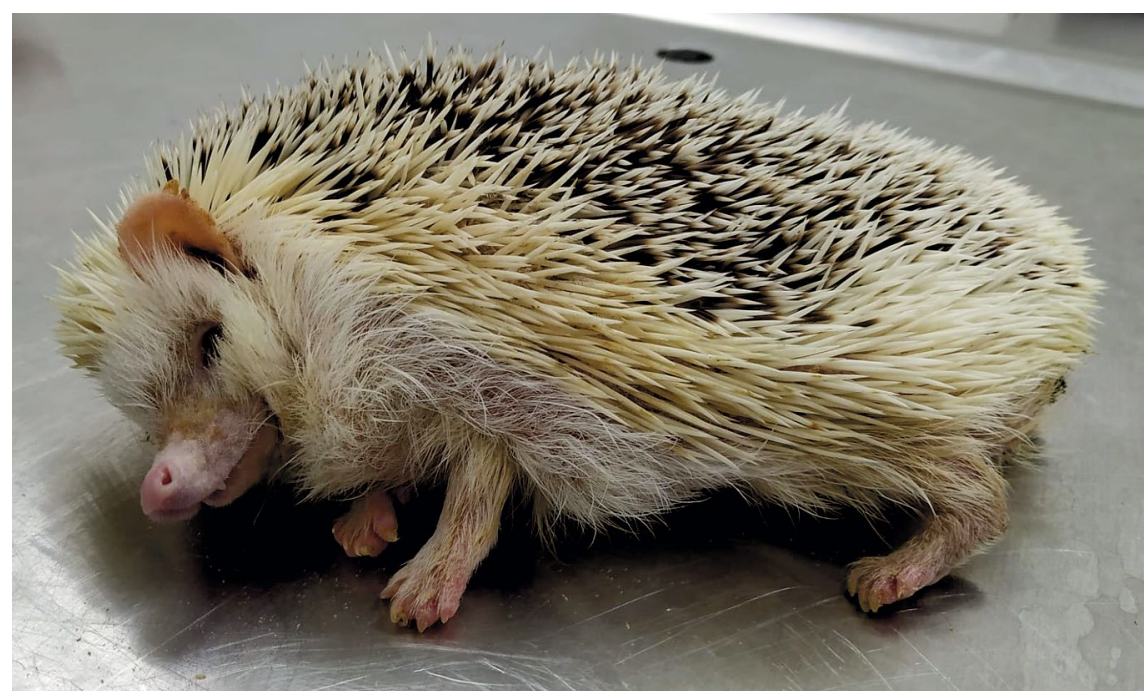

Fig. 1. African Pygmy Hedgehog with advanced neurological signs affecting four limbs (fot. J. Ruszkowski)

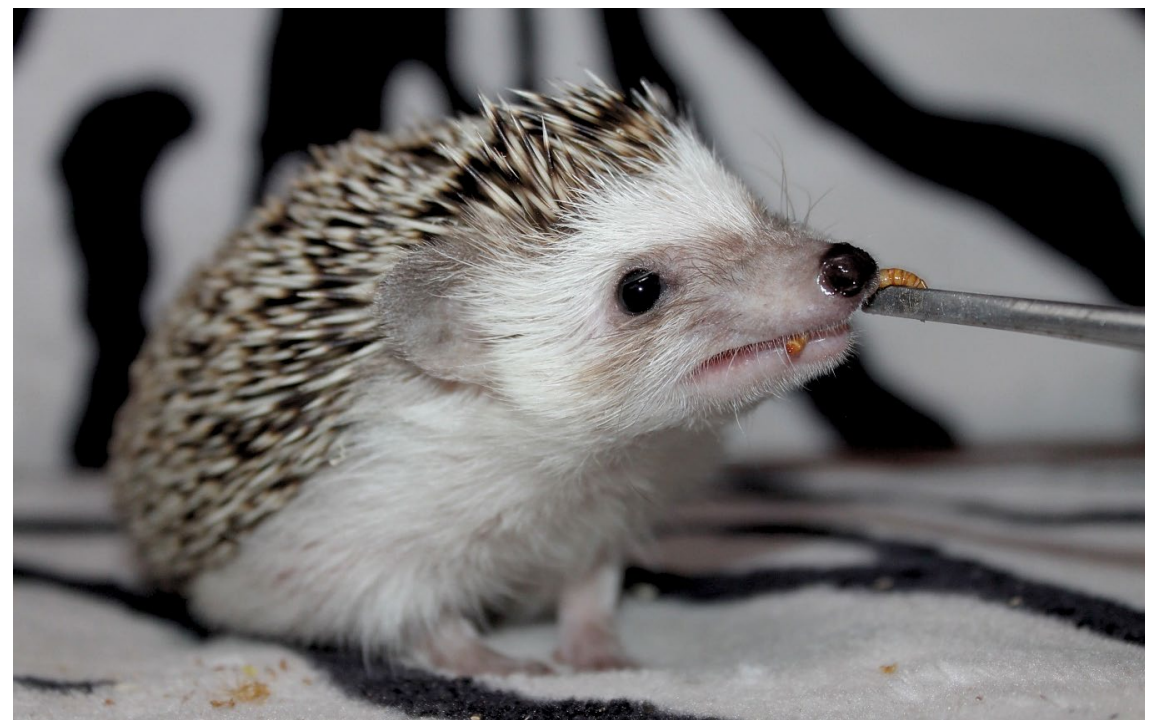

Fig. 2. Hedgehogs with neurological diseases may need assisted feeding to reach the pray easier (Fot. K. Wawoczna)

Hedgehogs: axonal loss, astrocytosis and microgliosis (15). Lesions were bilateral and symmetrical $(2,7)$. Similar, but smaller lesions of vacuolization of the white matter were also found in two asymptomatic hedgehogs (2). There were many concomitant diseases found during necropsies. One of the most frequent was mild to severe hepatic lipidosis. Nephritis, polycystic kidney disease and obesity were also reported $(2,7$, $11,12,16,17)$. Animals which had their blood tested, had higher alanine aminotransaminase, aspartate aminotransferase, total bilirubin and albumin values compared with references, which may suggest inappropriate diet and liver dysfunctions (16). Hedgehogs in captivity are often fed with mealworms (larvae of Tenebrio molitor) and dry cat food, both of which have much more fat and protein content than is recommended for hedgehogs $(1,3)$. Dry cat food may also be too hard to chew for a hedgehog, and may be irritating for its gingiva. This may lead to severe gingivitis and mucosal overgrowth. Intake of food containing
Tab. 1. The hematologic and serum biochemical values of hedgehogs

\begin{tabular}{|l|c|}
\hline PCV (\%) & $22-64$ \\
\hline RBC (10 $/ \mathrm{mL})$ & $3-16$ \\
\hline Hb (g/dL) & $7.0-21.1$ \\
\hline MCV (fL) & $41-94$ \\
\hline MCH (pg) & $11-31$ \\
\hline MCHC (g/dL) & $17-48$ \\
\hline Platelets (103/mL) & $60-347$ \\
\hline WBC (10 $/ \mathrm{mL})$ & $3-43$ \\
\hline Alkaline phosphatase (IU/L) & $8-42$ \\
\hline ALT (IU/L) & $16-134$ \\
\hline Amylase (IU/L) & $244-858$ \\
\hline AST (IU/L) & $8-137$ \\
\hline Bilirubin, total (mg/dL) & $0.0-1.3$ \\
\hline BUN (mg/dL) & $13-54$ \\
\hline Cholesterol (mg/dL) & $86-189$ \\
\hline Creatinine (mg/dL) & $0.0-0.8$ \\
\hline Phosphorus (mg/dL) & $2.4-12$ \\
\hline Protein, total (g/dL) & $4.0-7.7$ \\
\hline Albumin (g/dL) & $1.8-4.2$ \\
\hline
\end{tabular}

excessive amounts of fat and protein may lead to hepatic lipidosis and renal failure.

Both renal and hepatic dysfunctions may lead to changes in various organs, including the central nervous system (5). Some authors state that ischemia (which also may be caused by hepatic vasculopathy and cardiomyopathy) may be a cause of vacuolizations in CNS (5).

Renal failure can also be connected with cardiac problems. Cardiomyopathy is likewise a frequent finding in hedgehogs (17).

Moreover, in one mentioned study, a group of hedgehogs kept on wood shavings showed an increased number of pulmonary disorders, which may suggest an influence of wood dust on the respiratory tract. Wood shavings were also reported as a foreign body in the stomach that led to the death of two hedgehogs (16). Those examples show that shaved wood may not be a proper litter for hedgehogs and other small mammals.

An increasing number of hedgehog patients may be challenging for exotic pet veterinarians. Many health issues seem to be linked with each other and the symptoms may overlap in different diseases. Housing requirements like bedding type, available toys and even type of food for hedgehogs may differ depending on the source of information $(3,18)$. Incorrect housing may be a predisposing factor for many diseases. Many pathological findings may also depend on housing standards. Hedgehogs as pets are usually fed with dry cat food or mealworms, which may not meet their 
nutritional requirements, due to a high amount of protein and fat (1). Nowadays, with different types of commercial hedgehog food and availability of insects bred for food a better diet containing less protein and fat is recommended by clinicians (3). Wild hedgehogs eat a variety of insects, invertebrates, small mammals and plants, which make their diet much more diverse (3). Feeding supplements to insects given to hedgehogs may help to keep minerals and vitamins at a proper level (10). Correct, absorptive and dust-free bedding is also crucial for health of respiratory tract. Improper housing and diet may lead to the most common health problems in hedgehogs. Hepatic lipidosis, as well as renal and cardiac failure, may be the result of all those factors as well as may be linked with each other, making proper housing a very important means of disease prevention in pet hedgehogs. Those diseases may also be factors predisposing to neoplasms or WHS. Because of the nature of hedgehogs and their ability to hide symptoms during stressful situations like a visit to a veterinary clinic, it is important to perform a proper anamnesis. Some hedgehog patients have to be sedated to perform a clinical examination, which makes it not possible to see some symptoms. The owner should be asked in detail about hedgehog's housing, diet, any abnormalities in eating, moving, breathing or behavior. A correct clinical examination and blood collection for a check-up may be helpful in early diagnostics of the diseases. In clinical examination attention should be paid to palpation of the abdominal cavity in search for abnormalities in kidneys and tumors. The oral cavity should also be examined and checked for pathological changes, including masses growing on a palate and gingivas or dental problems. Ataxia, lack of appetite, weight loss and uncoordinated movements should be alarming for the veterinarian and further diagnostics should be performed. Performing blood tests (Tab. 1) and ultrasound examination should be advised to an owner on a check-up appointment. Kidneys and liver are organs that should be checked precisely, as some renal and hepatic dysfunctions were frequently found.

The knowledge about diseases in hedgehogs is still developing. Many diseases are still underdiagnosed, so establishing diagnostic protocols for pet hedgehogs is very important. Early diagnosed diseases are much easier to treat or monitor. It is also very important to provide to a hedgehog a healthy diet and proper environment.

\section{References}

1. Bernard J. B., Allen M. E.: Feeding captive insectivorous animals: nutritional aspects of insects as food, [in:] Nutrition Advisory Handbook Fact Sheet 003, 1997.

2. Díaz-Delgado J., Whitley D. B., Storts R. W., Heatley J. J., Hoppes S., Porter B. F.: The Pathology of Wobbly Hedgehog Syndrome. Vet. Pathol. 2018, 55, 711-718

3. Dierenfeld E. S.: Feeding Behavior and Nutrition of the African Pygmy Hedgehog (Atelerix albiventris). Vet. Clin. N. Am.: Exot. Anim. Prac. 2009, $12,335-337$

4. Garner M., Acvp D., Graesser D.: Wobbly Hedgehog Syndrome: A Neurodegenerative Disease of African and European Hedgehogs. Proceedings 2006.
5. Gill P. A., Townsend W. L.: Hepatic vasculopathy and encephalopathy in Brahman-type calves. Aust. Vet. J. 1993, 70, 69-69.

6. Glaser G. H.: Metabolic Encephalopathy in Hepatic, Renal and Pulmonary Disorders. Postgraduate Medicine 1960, 27, 611-619.

7. Graesser D., Spraker T. R., Dressen P., Garner M. M., Raymond J. T., Terwilliger G., Madri J. A.: Wobbly Hedgehog Syndrome in African Pygmy Hedgehogs (Atelerix spp.). J. Exotic Pet. Med. 2006, 15, 59-65.

8. Heatley J., Mauldin G., Cho D.: A Review of Neoplasia in the Captive African Hedgehog. Sem. Avian Exotic Pet. Med. 2005, 14, 182-192.

9. Karkamo V., Dillard K., Schulman K., Anttila M.: The Wobbly Hedgehog Syndrome - a clinical entity with variable CNS lesions. J. Comp. Pathol. 2013, 148, 96.

10. Klasing K. C., Thacker P., Lopez M. A., Calvert C. C.: Increasing the calcium content of mealworms (Tenebrio molitor) to improve their nutritional value for bone mineralization of growing chicks. J. Zoo Wildlife Med. 2000, 31, 512-518

11. Marciniak M., Skomorucha L.: Pierwszy w Polsce przypadek zespołu chwiejącego się jeża u jeża afrykańskiego. Życie Wet. 2012, 87, 1027-102.

12. Muñoz-Gutiérrez J. F., Garner M. M., Kiupel M.: Primary central nervous system neoplasms in African hedgehogs. J. Vet. Diagn. Invest. 2018, 30, 715-720.

13. Nakata M., Miwa Y., Itou T., Uchida K., Nakayama H., Sakai T.: Astrocytoma in an African Hedgehog (Atelerix albiventris) Suspected Wobbly Hedgehog Syndrome. J. Vet. Med. Sci. 2011, 73, 1333-1335.

14. Ogihara K., Suzuki K., Madarame H.: Primary Histiocytic Sarcoma of the Brain in an African Hedgehog (Atelerix albiventris). J. Comp. Pathol. 2017, 157, 241-245.

15. Palmer A. C., Blakemore W. F., Franklin R. J. M., Frost L. M., Gough R. E. Lewis J. C. M., Stocker L. R.: Paralysis in hedgehogs (Erinaceus europaeus) associated with demyelination. Vet. Rec. 1998, 143, 550-552.

16. Pei-Chi H., Jane-Fang Y., Lih-Chiann W. A.: Retrospective Study of the Medical Status on 63 African Hedgehogs (Atelerix albiventris) at the Taipei Zoo From 2003 to 2011. J. Exotic Pet. Med. 2015, 24, 105-111.

17. Raymond J. T., Garner M. M.: Cardiomyopathy in Captive African Hedgehogs (Atelerix Albiventris). J. Vet. Diagn. Invest. 2000, 12, 468-472.

18. Smith A. J.: Husbandry and Nutrition of Hedgehogs. Vet. Clin. N. Am. Exot. Anim. Pract. 1999, 2, 127-141.

19. Tynes V. V.: Hedgehogs, [in:] Tynes V. V. (ed): Behavior of Exotic Pets. WileyBlackwell, Ames I.A. 2010, p. 168-180

Corresponding author: Jakub Ruszkowski DVM, ul. Wojska Polskiego 71C, 60-625 Poznań, Poland; e-mail: ruszkowskijj@gmail.com 